\title{
Multiple Positive Solutions of Nonlinear Boundary Value Problem for Finite Fractional Difference
}

\author{
Yansheng He, Mingzhe Sun, and Chengmin Hou \\ Department of Mathematics, College of Science, Yanbian University, Yanji 133002, China \\ Correspondence should be addressed to Chengmin Hou; houchengmin@aliyun.com
}

Received 7 June 2014; Accepted 22 September 2014; Published 12 November 2014

Academic Editor: Patricia J. Y. Wong

Copyright (C) 2014 Yansheng He et al. This is an open access article distributed under the Creative Commons Attribution License, which permits unrestricted use, distribution, and reproduction in any medium, provided the original work is properly cited.

We consider a discrete fractional nonlinear boundary value problem in which nonlinear term $f$ is involved with the fractional order difference. And we transform the fractional boundary value problem into boundary value problem of integer order difference equation. By using a generalization of Leggett-Williams fixed-point theorem due to Avery and Peterson, we provide sufficient conditions for the existence of at least three positive solutions.

\section{Introduction}

Let $\mathbb{R}$ and $\mathbb{Z}$ be the sets of real numbers and integers, respectively. For $a, b \in \mathbb{R}, N \in \mathbb{Z}^{+}$with $b=a+N$, define $[a, b]_{\mathbb{N}_{a}}=\{a, a+1, \ldots, b\}$. Assume that $T$ is a given positive integer with $T>2$. We consider the fractional difference boundary value problem (briefly FBVP) of the forms

$$
\begin{gathered}
\Delta\left(\varphi_{p}\left(\Delta_{\nu-1}^{\nu} x(t)\right)\right)+q(t) \\
\cdot f\left(t+\nu-1, x(t+\nu-1),{ }_{t+\nu-\beta} \Delta_{\nu-1}^{\beta} x(t)\right)=0, \\
t \in[0, T]_{\mathbb{N}_{0}}, \\
{\left[\Delta_{\nu-1}^{\varepsilon} x(t)\right]_{t=T+1+\nu-\varepsilon}=0,} \\
x(\nu-1)=\eta\left[\Delta_{\nu-1}^{\delta} x(t)\right]_{t=\xi+1+\nu-\delta},
\end{gathered}
$$

where $\eta \in[0,1], \nu, \beta, \varepsilon, \delta \in(0,1)$ with $\beta, \delta \leq \nu \leq \varepsilon$, and $\varphi_{p}(s)=|s|^{p^{-2}} s, p>1, \xi \in[0, T]_{\mathbb{N}_{0}}, \Delta_{\nu-1}^{\varrho}(\varrho=\nu, \beta, \varepsilon, \delta)$ is fractional difference operator. We give the following assumptions:

(H1) $f(t+v-1, \cdot, \cdot):[\nu-1, T+\nu-1]_{\mathbb{N}_{v-1}} \times[0,+\infty) \times \mathbb{R} \rightarrow$ $[0,+\infty)$ is continuous;

(H2) $q(t)$ is nonnegative on $[0, T]_{\mathbb{N}_{0}}, q(t) \equiv 0$ does not hold on $[0, T]_{\mathbb{N}_{0}}$, and $\sum_{t=0}^{T} q(t)<\infty$.
Fixed-point theorems and their applications in nonlinear problems have a long history, some of which are documented in Zeidler's book [1]. There seems to be increasing interest in multiple fixed-point theorems and their applications to boundary value problems for ordinary differential equations or finite difference equations. The applications can be found in the papers [2-6]. An interest in triple solutions has evolved from the Leggett-Williams multiple fixed-point theorem [7]. And, lately, two triple fixed-point theorems by Avery and Peterson [4] and Avery [8] have been applied to obtain triple solutions of certain boundary value problems for ordinary differential equations as well as for their discrete analogues. On the other hand, fractional differential and difference "operators" are found themselves in concrete applications, and hence attention has to be paid to associated fractional difference and differential equations under various boundary or side conditions. For example, Atici and Eloe [9] explored some of the theories of a discrete conjugate FBVP in [9]. Similarly, in [10], a discrete right-focal FBVP was analyzed. Other recent advances in the theory of the discrete fractional calculus may be found in [11-26]. In particular, an interesting recent paper by Atici and Şengül [14] addressed the use of fractional difference equations in tumor growth modeling. Thus, it seems that there exists some promise in using fractional difference equations as mathematical models for describing physical problems in more accurate manners. 
In order to handle the existence problem for FBVP, various methods (among which are some standard fixedpoint theorems) can be used. For example, in [10, 12, 27], authors investigated the existence to some boundary value problems by fixed-point theorems on a cone. In [28], we established the existence conditions for a boundary value problem by using the coincidence degree theory. In [29], authors pointed out the existence of multiple solutions for a FBVP with parameter by establishing the corresponding variational framework and using the mountain pass theorem, linking theorem, and Clark theorem in critical point theory. To the best of our knowledge, Leggett-Williams fixed-point theorem has not been used in discrete fractional boundary value problems. The aim of this paper is to establish the existence conditions for boundary value problem (1)-(2). The proof relies on the Leggett-Williams fixed-point theorem.

Throughout this paper, we make the convention that $\sum_{i=j}^{m} x(i)=0$ for $m<j$ and denote $\varphi_{q}=\varphi_{p}^{-1}, 1 / p+1 / q=1$.

\section{Preliminaries}

In this section, we collect some basic definitions and lemmas for manipulating discrete fractional operators. These and other related results can be found in $[4,12,14,16]$.

For any integer $\beta$, let $\mathbb{N}_{\beta}=\{\beta, \beta+1, \beta+2, \ldots\}$. We define $t^{(v)}:=\Gamma(t+1) / \Gamma(t+1-v)$, for any $t$ and $\nu$ for which the righthand side is defined. We also appeal to the convention that if $t+1-v$ is a pole of the Gamma function and $t+1$ is not a pole, then $t^{(\nu)}=0$.

Definition 1. The $v$ th fractional sum of $f$ for $v>0$ is defined by

$$
\Delta_{a}^{-\nu} f(t)=\frac{1}{\Gamma(\nu)} \sum_{s=a}^{t-v}(t-s-1)^{(\nu-1)} f(s),
$$

for $t \in \mathbb{N}_{a+v}$. We also define the $v$ th fractional difference for $\nu>0$ by $\Delta_{a}^{v} f(t):=\Delta^{N} \Delta_{a}^{\nu-N} f(t)$, where $t \in \mathbb{N}_{a+N-v}$, and $N \in \mathbb{N}$ is chosen so that $0 \leq N-1<\nu \leq N$.

Definition 2. Let $\mathbb{X}$ be a real Banach space. A nonempty closed convex set $P \subset \mathbb{X}$ is called a cone of $\mathbb{X}$ if it satisfies the following conditions:

(1) $x \in P, \lambda \geq 0$ implies $\lambda x \in P$;

(2) $x \in P,-x \in P$ implies $x=0$.

Every cone $P \subset \mathbb{X}$ induces a partial ordering “ $\leq$ ” on $\mathbb{X}$ defined by $x \leq y$ if and only if $y-x \in P$.

Definition 3. Given a cone $P$ in a real Banach space $\mathbb{X}$, a functional $\psi: P \rightarrow \mathbb{R}$ is said to be increasing on $P$, provided that $\psi(x) \leq \psi(y)$ for all $x, y \in P$ with $x \leq y$.

Let $\gamma$ and $\theta$ be nonnegative continuous convex functionals on $P, \alpha$ a nonnegative continuous concave functional on $P$, and $\psi$ a nonnegative continuous functional on $P$. Then, for positive real numbers $a, b, c$, and $d$, we define the following convex sets:

$$
\begin{aligned}
& P(\gamma, d)=\{x \in P \mid \gamma(x)<d\}, \\
& P(\gamma, \alpha, b, d)=\{x \in P \mid b \leq \alpha(x), \gamma(x) \leq d\}, \\
& P(\gamma, \theta, \alpha, b, c, d)=\{x \in P \mid b \leq \alpha(x), \theta(x) \leq c, \gamma(x) \leq \\
& d\} \text { and a closed set } R(\gamma, \psi, a, d)=\{x \in P \mid a \leq \\
& \psi(x), \gamma(x) \leq d\} .
\end{aligned}
$$

The following fixed-point theorem due to Avery and Peterson is fundamental in the proof of our main results.

Lemma 4 (see [4]). Let $\mathbb{X}$ be a Banach space and let $P \subset \mathbb{X}$ be a cone. Let $\gamma$ and $\theta$ be nonnegative continuous convex functionals on $P, \alpha$ a nonnegative continuous concave functional on $P$, and $\psi$ a nonnegative continuous functional on $P$ satisfying $\psi(\lambda x) \leq \lambda \psi(x)$ for $0 \leq \lambda \leq 1$, such that, for some positive numbers $M$ and $d, \alpha(x) \leq \psi(x)$ and $\|x\| \leq M \gamma(x)$ for all $x \in \overline{P(\gamma, d)}$. Suppose $Q: \overline{P(\gamma, d)} \rightarrow \overline{P(\gamma, d)}$ is completely continuous and there exist positive numbers $a, b, c$ with $a<b$ such that

(S1) $\{x \in P(\gamma, \theta, \alpha, b, c, d) \mid \alpha(x)>b\} \neq \emptyset$ and $\alpha(Q x)>b$ for $x \in P(\gamma, \theta, \alpha, b, c, d)$;

(S2) $\alpha(Q x)>b$ for $x \in P(\gamma, \alpha, b, d)$ with $\theta(Q x)>c$;

(S3) $0 \notin R(\gamma, \psi, a, d)$ and $\psi(Q x)<a$ for $x \in R(\gamma, \psi, a, d)$ with $\psi(x)=a$

Then $Q$ has at least three fixed points $x_{1}, x_{2}, x_{3} \in \overline{P(\gamma, d)}$ such that

$$
\begin{aligned}
& \gamma\left(x_{i}\right) \leq d \text { for } i=1,2,3 \\
& b<\alpha\left(x_{1}\right), \psi\left(x_{3}\right)<a, a<\psi\left(x_{2}\right) \text { with } \alpha\left(x_{2}\right)<b .
\end{aligned}
$$

Lemma 5 (see [16]). Let $y: \mathbb{N}_{a} \rightarrow \mathbb{R}$ and $\nu>0$ with $N-1<$ $\nu \leq N$, and then

$$
\Delta_{a}^{v} y(t)=\sum_{k=0}^{t-a+v}(-1)^{k}\left(\begin{array}{l}
v \\
k
\end{array}\right) y(t+v-k),
$$

for $t \in \mathbb{N}_{a+N-v}$, where $\left(\begin{array}{l}v \\ k\end{array}\right)=\Gamma(\nu+1) / \Gamma(k+1) \Gamma(v-k+1)$.

Lemma 6 (see [16]). Let $f: \mathbb{N}_{a} \rightarrow \mathbb{R}$ be given and suppose $k \in \mathbb{N}_{0}$ and $v>0$. Then, for $t \in \mathbb{N}_{a+v}$,

$$
\begin{aligned}
& \Delta_{a}^{-v} \Delta^{k} f(t) \\
& \quad=\Delta_{a}^{k-v} f(t)-\sum_{j=0}^{k-1} \frac{\Delta^{j} f(a)}{\Gamma(\nu-k+j+1)}(t-a)^{(\nu-k+j)} .
\end{aligned}
$$

Moreover, if $\mu>0$ with $M-1 \leq \mu \leq M$, then, for $t \in$ $\mathbb{N}_{a+M-\mu+\nu}$,

$$
\begin{aligned}
& \Delta_{a+M-\mu}^{-v} \Delta_{a}^{\mu} f(t) \\
& =\Delta_{a}^{\mu-v} f(t)-\sum_{j=0}^{M-1} \frac{\Delta_{a}^{j-M+\mu} f(a+M-\mu)}{\Gamma(\nu-M+j+1)} \\
& \cdot(t-a-M+\mu)^{(\nu-M+j)} .
\end{aligned}
$$


Lemma 7 (see [16]). Let $f: \mathbb{N}_{a} \rightarrow \mathbb{R}$ be given and suppose $\mu, \nu>0$ with $N-1<\mu \leq N$. Then, for $t \in \mathbb{N}_{a+\nu+N-\mu}$,

$$
\Delta_{a+v}^{\mu} \Delta_{a}^{-v} f(t)=\Delta_{a}^{\mu-v} f(t) .
$$

Lemma 8 (see [16]). Let $f: \mathbb{N}_{a} \rightarrow \mathbb{R}$ be given and suppose $v>0$ with $N-1<v<N$. Then, for $t \in \mathbb{N}_{a-v+N}$,

$$
\begin{aligned}
& \Delta_{a}^{v} f(t) \\
& \quad= \begin{cases}\frac{1}{\Gamma(-v)} \sum_{s=a}^{t+v}(t-s-1)^{(-v-1)} f(s), & N-1<\nu<N ; \\
\Delta^{N} f(t), & \nu=N .\end{cases}
\end{aligned}
$$

Lemma 9 (see $[16]$ ). Let $a \in \mathbb{R}$ and $\mu>0$ be given. Then,

$$
\Delta(t-a)^{(\mu)}=\mu(t-a)^{(\mu-1)},
$$

for anyt for which both sides are well-defined. Furthermore, for $v>0$,

$$
\begin{gathered}
\Delta_{a+\mu}^{-v}(t-a)^{(\mu)}=\mu^{(-\nu)}(t-a)^{(\mu+\nu)}, \text { for } t \in \mathbb{N}_{a+\mu+\nu}, \\
\Delta_{a+\mu}^{\nu}(t-a)^{(\mu)}=\mu^{(\nu)}(t-a)^{(\mu-\nu)}, \text { for } t \in \mathbb{N}_{a+\mu+N-v} .
\end{gathered}
$$

\section{Triple Positive Solutions}

In this section, we impose growth conditions on $f$ to obtain the triple positive solutions for FBVP (1)-(2).

For the sake of convenience, for $0<\delta \leq \nu \leq \varepsilon, \xi \in$ $[0, T]_{\mathbb{N}_{0}}, \eta \in[0,1], p>1$, we set the following notations:

$$
\begin{aligned}
& \kappa(t, \rho) \\
& =\frac{(t+1+\nu-\rho)^{(\nu-\rho-1)}}{\Gamma(\nu-\rho)}, \quad \text { for }(t, \rho) \in[-2, T]_{\mathbb{N}_{-2}} \times(0,1) \text {, } \\
& \tau=-\sum_{s=0}^{T} \kappa(T-s-1, \varepsilon)-\frac{\eta \kappa(T, \varepsilon)}{1-\eta \kappa(\xi, \delta)} \\
& \cdot \sum_{s=0}^{\xi+1} \kappa(\xi-s-1, \delta) \\
& M_{1}=\frac{\sum_{i=0}^{T} q(i)}{1-\tau^{p-1}} \\
& M_{2}=\frac{\eta}{1-\eta \kappa(\xi, \delta)} \sum_{s=0}^{\xi+1} \kappa(\xi-s-1, \delta) \varphi_{q}\left(\sum_{j=s}^{T} q(j)\right), \\
& M_{3}=\sum_{j=0}^{T} \varphi_{q}\left(\sum_{i=j}^{T} q(i)\right) \\
& N=\max \left\{M_{2}, M_{3}\right\}, \\
& m=\frac{\eta(\xi+2)(\xi+1+\nu-\delta)^{(\nu-\delta-1)}}{(1-\eta \kappa(\xi, \delta)) \Gamma(\nu-\delta+1)} .
\end{aligned}
$$

Then, from (11), we immediately obtain some properties of $\kappa(t, \varrho)$ as follows:

$$
\kappa(t, \varrho) \in \begin{cases}\{1\}, & (t, \varrho) \in\{-2\} \times(0,1), \\ (0,1), & (t, \varrho) \in[-1, T]_{\mathbb{N}_{-1}} \times(0, \nu), \\ \{0\}, & (t, \varrho) \in[-1, T]_{\mathbb{N}_{-1}} \times\{v\}, \\ (-1,0), & (t, \varrho) \in[-1, T]_{\mathbb{N}_{-1}} \times(\nu, 1) .\end{cases}
$$

We will also need the following elementary facts:

$$
\tau \in \begin{cases}\{0\}, & \delta \leq v=\varepsilon, \\ (0,1), & \delta \leq \nu<\varepsilon .\end{cases}
$$

Indeed, if $\delta \leq \nu=\varepsilon$, then, by (12) and (14), clearly, $\tau=0$. If $\delta=v<\varepsilon$, then

$$
\begin{aligned}
& \tau=\left(-\sum_{s=0}^{T} \kappa(T-s-1, \varepsilon)-\eta \kappa(T, \varepsilon)\right) \\
& =1-\sum_{s=0}^{T+1} k(T-s-1, \varepsilon)-\eta \kappa(T, \varepsilon) \\
& =1-\frac{1}{\Gamma(\nu-\varepsilon)} \sum_{s=0}^{T+1}(T+\nu-\varepsilon-s)^{(\nu-\varepsilon-1)}-\eta \kappa(T, \varepsilon) \\
& =1-\left(1+(\nu-\varepsilon)+\frac{(1+\nu-\varepsilon)(\nu-\varepsilon)}{2 !}\right. \\
& \left.+\cdots+\frac{(T+\nu-\varepsilon) \cdots(\nu-\varepsilon)}{(T+1) !}\right)-\eta \kappa(T, \varepsilon) \\
& =1-\left((1+\nu-\varepsilon)\left(1+\frac{\nu-\varepsilon}{2}\right)+\frac{(2+\nu-\varepsilon) \cdots(\nu-\varepsilon)}{3 !}\right. \\
& \left.+\cdots+\frac{(T+\nu-\varepsilon) \cdots(\nu-\varepsilon)}{(T+1) !}\right)-\eta \kappa(T, \varepsilon) \\
& =1-\left(\frac{(1+\nu-\varepsilon)(2+\nu-\varepsilon)}{2 !}\left(1+\frac{\nu-\varepsilon}{3}\right)\right. \\
& \left.+\cdots+\frac{(T+\nu-\varepsilon) \cdots(\nu-\varepsilon)}{(T+1) !}\right)-\eta \kappa(T, \varepsilon) \\
& =\cdots \\
& =1-\left(\frac{(T+1+\nu-\varepsilon)(T+\nu-\varepsilon) \cdots(1+\nu-\varepsilon)}{(T+1) !}\right) \\
& -\eta \frac{(T+1+\nu-\varepsilon)(T+\nu-\varepsilon) \cdots(\nu-\varepsilon)}{(T+2) !} \\
& =1-\left(\frac{(T+1+\nu-\varepsilon)(T+\nu-\varepsilon) \cdots(1+\nu-\varepsilon)}{(T+1) !}\right) \\
& \cdot\left(1+\eta \frac{\nu-\varepsilon}{T+2}\right) \\
& =1-\frac{(T+2) \kappa(T, \varepsilon)}{\nu-\varepsilon}\left(1-\eta \frac{\varepsilon-\nu}{T+2}\right) \text {. }
\end{aligned}
$$

It follows that $0<\tau<1$. 
If $\delta<\nu<\varepsilon$, then, since

$$
\begin{aligned}
& \left(1+\frac{(\varepsilon-\nu)(\xi+2)}{(\nu-\delta)(T+2)}\right) \kappa(\xi, \delta) \\
& \leq\left(1+\frac{\varepsilon-\nu}{\nu-\delta}\right) \kappa(\xi, \delta) \\
& =\frac{(\varepsilon-\delta)(\xi+1+\nu-\delta)^{(\nu-\delta-1)}}{\Gamma(\nu-\delta+1)} \\
& =(\varepsilon-\delta) \frac{\Gamma(\xi+2+\nu-\delta)}{\Gamma(\xi+3) \Gamma(\nu-\delta+1)} \\
& =(\varepsilon-\delta) \frac{(\xi+1+\nu-\delta) \cdots(\nu-\delta+1)}{(\xi+2) \cdots 2} \\
& <1 \text {, } \\
& \tau=-\sum_{s=0}^{T} \kappa(T-s-1, \varepsilon)-\frac{\eta \kappa(T, \varepsilon)}{(1-\eta \kappa(\xi, \delta))} \\
& \cdot \sum_{s=0}^{\xi+1} \kappa(\xi-s-1, \delta) \\
& =1-\sum_{s=0}^{T+1} \kappa(T-s-1, \varepsilon)-\frac{\eta \kappa(T, \varepsilon)}{(1-\eta \kappa(\xi, \delta))} \\
& \cdot \sum_{s=0}^{\xi+1} \kappa(\xi-s-1, \delta) \\
& =1-\frac{(T+2) \kappa(T, \varepsilon)}{\nu-\varepsilon}-\frac{\eta \kappa(T, \varepsilon) \kappa(\xi, \delta)(\xi+2)}{(1-\eta \kappa(\xi, \delta))(\nu-\delta)} \\
& =1-\frac{(T+2) \kappa(T, \varepsilon)}{\nu-\varepsilon} \\
& \cdot\left(1-\frac{(\varepsilon-\nu)}{(\nu-\delta)} \frac{\eta(\xi+2) \kappa(\xi, \delta)}{(1-\eta \kappa(\xi, \delta))(T+2)}\right) \\
& =1-\frac{(T+2) \kappa(T, \varepsilon)}{\nu-\varepsilon} \\
& \cdot\left(\left(1-\eta \kappa(\xi, \delta)-\frac{(\varepsilon-\nu)(\xi+2)}{(\nu-\delta)(T+2)} \eta \kappa(\xi, \delta)\right)\right. \\
& \left.\cdot(1-\eta \kappa(\xi, \delta))^{-1}\right) \\
& =1-\frac{(T+2) \kappa(T, \varepsilon)}{\nu-\varepsilon} \\
& \cdot \frac{1-(1+((\varepsilon-\nu)(\xi+2) /(\nu-\delta)(T+2))) \eta \kappa(\xi, \delta)}{1-\eta \kappa(\xi, \delta)} .
\end{aligned}
$$

We have $0<\tau<1$.
Now, suppose that $x(t)$ is a solution of the problem (1)-(2), and let $y(t)=\Delta_{\nu-1}^{-(1-\gamma)} x(t)$. Then, from Lemmas 6, 7, 8, and 9, we have $x(t)=\Delta_{0}^{1-v} y(t)=\Delta_{0}^{-v} \Delta y(t)+(y(0) / \Gamma(\nu)) t^{(\nu-1)}$, and

$$
\begin{aligned}
{ }_{t+\nu-\beta} \Delta_{\nu-1}^{\beta} x(t) & \\
= & { }_{t+\nu-\beta} \Delta_{\nu-1}^{\beta}\left(\Delta_{0}^{-v} \Delta y(t)\right)+\frac{y(0)}{\Gamma(\nu)}{ }_{t+\nu-\beta} \Delta_{\nu-1}^{\beta} t^{(\nu-1)} \\
= & \frac{1}{\Gamma(-\beta)}\left(\sum_{s=\nu}^{t+v}(t+\nu-\beta-s-1)^{(-\beta-1)} \Delta_{0}^{-v} \Delta y(s)\right. \\
& \left.+\left.(t+\nu-\beta-s-1)^{(-\beta-1)} \Delta_{0}^{-v} \Delta y(s)\right|_{s=\nu-1}\right) \\
& +\frac{y(0)}{\Gamma(\nu)}(\nu-1)^{(\beta)}(t+\nu-\beta)^{(\nu-\beta-1)} \\
= & { }_{t+\nu-\beta} \Delta_{\nu}^{\beta} \Delta_{0}^{-v} \Delta y(t)+\frac{y(0)}{\Gamma(\nu-\beta)}(t+\nu-\beta)^{(\nu-\beta-1)} \\
= & { }_{t+\nu-\beta} \Delta_{0}^{-(\nu-\beta)} \Delta y(t)+\frac{y(0)}{\Gamma(\nu-\beta)}(t+\nu-\beta)^{(\nu-\beta-1)} \\
= & \sum_{s=0}^{t} \kappa(t-s-2, \beta) \Delta y(s)+y(0) \kappa(t-1, \beta) .
\end{aligned}
$$

Similarly, we have

$$
\begin{aligned}
& {\left[\Delta_{\nu-1}^{\varepsilon} x(t)\right]_{t=T+1+\nu-\varepsilon}} \\
& =\sum_{s=0}^{T+1} \kappa(T-s-1, \varepsilon) \Delta y(s)+y(0) \kappa(T, \varepsilon), \\
& {\left[\Delta_{\nu-1}^{\delta} x(t)\right]_{t=\xi+1+\nu-\delta}} \\
& =\sum_{s=0}^{\xi+1} \kappa(\xi-s-1, \delta) \Delta y(s)+y(0) \kappa(\xi, \delta) .
\end{aligned}
$$

Thus, by the transformation $y(t)=\Delta_{v-1}^{-(1-v)} x(t)$, the problem (1)-(2) is equivalent to the following problem (20)-(22):

$$
\begin{array}{r}
\Delta\left(\varphi_{p}(\Delta y(t))\right)+q(t) \\
\cdot f\left(t+v-1, x(t+v-1),{ }_{t+\nu-\beta} \Delta_{\nu-1}^{\beta} x(t)\right)=0, \\
t \in[0, T]_{\mathbb{N}_{0}},
\end{array}
$$

$\Delta y(T+1)$

$$
=-\sum_{s=0}^{T} \kappa(T-s-1, \varepsilon) \Delta y(s)-y(0) \kappa(T, \varepsilon),
$$

$y(0)$

$$
=\eta \sum_{s=0}^{\xi+1} \kappa(\xi-s-1, \delta) \Delta y(s)+\eta y(0) \kappa(\xi, \delta),
$$


where

$$
\begin{gathered}
x(t+\nu-1)=\Delta_{0}^{1-\nu} y(t+v-1), \\
{ }_{t+\nu-\beta} \Delta_{\nu-1}^{\beta} x(t) \\
=\sum_{s=0}^{t} \kappa(t-s-2, \beta) \Delta y(s)+y(0) k(t-1, \beta) .
\end{gathered}
$$

Now, suppose that $y(t)$ is a solution of (20)-(22). We will show that

$$
\begin{gathered}
y(t) \geq 0, \\
\Delta y(t) \geq 0, \\
\Delta^{2} y(t) \leq 0 .
\end{gathered}
$$

Firstly, it is easy to see that $\Delta^{2} y(t) \leq 0$ from $\Delta \varphi_{p}(\Delta y(t)) \leq 0$.

Secondly, if $\delta \leq \nu=\varepsilon$, then $\Delta y(T+1)=0$ by (21) and (22). If $\delta \leq v<\varepsilon$, we have

$$
\begin{aligned}
\Delta y & (T+1) \\
= & -\sum_{s=0}^{T} \kappa(T-s-1, \varepsilon) \Delta y(s) \\
& -\frac{\eta \kappa(T, \varepsilon)}{1-\eta \kappa(\xi, \delta)} \sum_{s=0}^{\xi+1} \kappa(\xi-s-1, \delta) \Delta y(s) \\
\geq & -\sum_{s=0}^{T} \kappa(T-s-1, \varepsilon) \\
& \left.\quad-\frac{\eta \kappa(T, \varepsilon)}{1-\eta \kappa(\xi, \delta)} \sum_{s=0}^{\xi+1} \kappa(\xi-s-1, \delta)\right) \Delta y(T+1) \\
= & \tau \Delta y(T+1) .
\end{aligned}
$$

So we obtain that $\Delta y(T+1) \geq 0$ and hence $\Delta y(t) \geq 0$ for $t \in[0, T+1]_{\mathbb{N}_{0}}$. Note that $y(0)=\left(\eta \sum_{s=0}^{\xi+1}(\kappa(\xi-s-\right.$ $1, \delta) \Delta y(s))) /(1-\eta \kappa(\xi, \delta)) \geq 0$. Thus (24) holds.

Next, clearly $y(t)=\Delta_{\nu-1}^{-(1-\nu)} x(t)=(1 / \Gamma(1-\nu)) \sum_{s=\gamma-1}^{t+\nu-1}(t-$ $s-1)^{(-\nu)} x(s) \geq 0$ if $x(t) \geq 0$.

On the other hand, if $y(t)$ is a solution of (20)-(22), then, from $x(t)=\Delta_{0}^{1-v} y(t)=\sum_{k=0}^{t+1-v}(-1)^{k}\left(\begin{array}{c}1-v \\ k\end{array}\right) y(t+1-v-k)$, we have

$$
\begin{aligned}
x(\nu-1) & =y(0) \geq 0, \\
y(1) & \geq x(\nu)=y(1)-(1-v) y(0) \geq v y(1),
\end{aligned}
$$

$$
\begin{aligned}
y(2) \geq & x(v+1)=y(2)-(1-v) y(1) \\
& +\frac{(1-v)(-v)}{2 !} y(0) \\
\geq & \frac{v(1+v)}{2 !} y(2), \\
& \vdots \\
y(t) \geq & x(t+v-1)=y(t)-(1-v) y(t-1) \\
& +\cdots+\frac{(1-v)(-v) \cdots(-v-t+2)}{t !} y(0) \\
\geq & \frac{\nu(1+v) \cdots(t+v-1)}{t !} y(t) \\
= & \frac{1}{\Gamma(\nu)}(t+v-1)^{(\nu-1)} y(t) \\
\geq & \frac{1}{\Gamma(\nu)}(T+v-1)^{(\nu-1)} y(t), \quad t \in[0, T]_{\mathbb{N}_{0}} ;
\end{aligned}
$$

that is,

$$
\frac{(T+\nu-1)^{(\nu-1)}}{\Gamma(\nu)} y(t) \leq x(t+\nu-1) \leq y(t), \quad t \in[0, T]_{\mathbb{N}_{0}} .
$$

This implies that the problem (1)-(2) has positive solutions if and only if the problem (20)-(22) has positive solutions. In the sequel, we will concretely consider the boundary problem (20)-(22).

Summing (20) from $t$ to $T$, we find that

$$
\begin{aligned}
\varphi_{p} & (\Delta y(T+1))-\varphi_{p}(\Delta y(t)) \\
& =-\sum_{i=t}^{T} q(i) f\left(i+\nu-1, x(i+\nu-1),{ }_{i+\nu-\beta} \Delta_{\nu-1}^{\beta} x(i)\right) .
\end{aligned}
$$

Thus,

$$
\begin{aligned}
& \Delta y(t) \\
& =\varphi_{q}\left(\sum_{i=t}^{T} q(i) f\left(i+\nu-1, x(i+\nu-1),{ }_{i+\nu-\beta} \Delta_{\nu-1}^{\beta} x(i)\right)\right. \\
& \left.\quad+\varphi_{p}(\Delta y(T+1))\right) .
\end{aligned}
$$


Summing (29) from 0 to $t-1$, it follows that

$$
\begin{aligned}
& y(t)-y(0) \\
& =\sum_{j=0}^{t-1} \varphi_{q}\left(\sum_{i=j}^{T} q(i) f\left(i+\nu-1, x(i+\nu-1),{ }_{i+\nu-\beta} \Delta_{\nu-1}^{\beta} x(i)\right)\right. \\
& \left.\quad+\varphi_{p}(\Delta y(T+1))\right)
\end{aligned}
$$

which, together with (22), implies that

$$
\begin{gathered}
y(t) \\
=\frac{\eta}{1-\eta \kappa(\xi, \delta)} \\
\cdot \sum_{s=0}^{\xi+1} \kappa(\xi-s-1, \delta) \varphi_{q} \\
\cdot\left(\sum_{j=s}^{T} q(j) f(j+\nu-1, x(j+\nu-1),\right. \\
\left.+\varphi_{p}(\Delta y(T+1))\right) \\
+\sum_{j=0}^{t-1} \varphi_{q}\left(\sum_{i=j}^{T} q(i) f(i+\nu-1, x(i+\nu-1),\right. \\
\left.+\varphi_{\nu-1}^{\beta} x(j)\right) \\
\left.i+\nu-\beta \Delta_{\nu-1}^{\beta} x(i)\right) \\
\\
\left.+\varphi_{p}(\Delta y(T+1))\right) .
\end{gathered}
$$

Next, let $B=\left\{y:[0, T+2]_{\mathbb{N}_{0}} \rightarrow \mathbb{R}\right\}$ be endowed with the norm $\|y\|=\max \left\{\|y\|_{\infty},\|\Delta y\|_{\infty}\right\}$, where $\|y\|_{\infty}=$ $\max _{t \in[0, T+2]_{N_{0}}}|y(t)|$. Choose the cone $P \subset B$ defined by

$$
\begin{aligned}
P=\left\{y \in B: y(t) \geq 0 \text { for } t \in[0, T+2]_{\mathbb{N}_{0}},\right. \\
\Delta y(t) \geq 0 \text { for } t \in[0, T+1]_{\mathbb{N}_{0}}, \\
\left.\Delta^{2} y(t) \leq 0, \text { for } t \in[0, T]_{\mathbb{N}_{0}} \text { and (21)(22) hold }\right\} .
\end{aligned}
$$

Let the nonnegative continuous concave functional $\alpha$, the nonnegative continuous convex functional $\theta, \gamma$, and the nonnegative continuous functional $\psi$ be defined on the cone $P$ by

$$
\begin{gathered}
\gamma(y)=\max _{t \in[0, T+1]_{N_{0}}}|\Delta y(t)|, \\
\alpha(y)=\min _{t \in[h, T+2]_{N_{0}}}|y(t)|, \\
\psi(y)=\theta(y)=\max _{t \in[0, T+2]_{N_{0}}}|y(t)|,
\end{gathered}
$$

where $h=[(T+2) / 2]$ and $[x]$ is the greatest integer not greater than $x$. Clearly, $\gamma(y)=\Delta y(0), \psi(y)=\theta(y)=y(T+2)$, and $\alpha(y)=y(h)$.

For $t \in[0, T+2]_{\mathbb{N}_{0}}$, define an operator $Q: P \rightarrow B$ by

$(Q y)(t)$

$$
\begin{aligned}
& =\frac{\eta}{1-\eta \kappa(\xi, \delta)} \sum_{s=0}^{\xi+1} \kappa(\xi-s-1, \delta) \varphi_{q} \\
& \cdot\left(\sum_{j=s}^{T} q(j) f\left(j+\nu-1, x(j+\nu-1),{ }_{j+\nu-\beta} \Delta_{\nu-1}^{\beta} x(j)\right)\right. \\
& \left.\quad+\varphi_{p}(\Delta y(T+1))\right) \\
& +\sum_{i=0}^{t-1} \varphi_{q}\left(\sum_{j=i}^{T} q(j)\right. \\
& \cdot f\left(j+\nu-1, x(j+\nu-1),_{j+\nu-\beta} \Delta_{\nu-1}^{\beta} x(j)\right) \\
& \left.+\varphi_{p}(\Delta y(T+1))\right) .
\end{aligned}
$$

We next require a preliminary lemma.

Lemma 10. Let $Q$ be defined by the above equation. If $y \in P$, then

(i) $\Delta(Q y)(t) \geq 0$ for $t \in[0, T+1]_{\mathbb{N}_{0}},(Q y)(t) \geq 0$ for $t \in[0, T+2]_{\mathbb{N}_{0}}$;

(ii) $\Delta(\mathrm{Q} y)(T+1)=-\sum_{s=0}^{T} \kappa(T-s-1, \varepsilon) \Delta(\mathrm{Q} y)(s)-$ $(\mathrm{Q} y)(0) \kappa(T, \varepsilon),(\mathrm{Q} y)(0)=(\eta /(1-\eta \kappa(\xi, \delta))) \sum_{s=0}^{\xi+1} \kappa(\xi-$ $s-1, \delta) \Delta(Q y)(s)$;

(iii) $Q: P \rightarrow P$ is completely continuous;

(iv) finding positive solutions of FBVP (20)-(22) is equivalent to finding fixed points of the operator $Q$ on $P$;

(v) If $y \in P, y(t) \geq(t /(T+2))\|y\|_{\infty}=(t /(T+2)) y(T+2)$, for $t \in[0, T+2]_{\mathbb{N}_{0}}$.

The proof is simple and omitted. 
By Lemma 10 and (33), for all $y \in \overline{P(\gamma, d)} \subset P$, the functionals defined above satisfy

$$
\frac{h}{T+2} \theta(y) \leq \alpha(y) \leq \theta(y)=\psi(y) .
$$

Furthermore, since

$$
\frac{y(T+2)-y(0)}{T+2} \leq \Delta y(0),
$$

$$
\begin{aligned}
y & (T+2) \\
& \leq y(0)+(T+2) \Delta y(0) \\
& \leq\left(\frac{\eta}{1-\eta \kappa(\xi, \delta)} \sum_{s=0}^{\xi+1} \kappa(\xi-s-1, \delta)+T+2\right) \Delta y(0) \\
& =\left(\frac{\eta(\xi+2)(\xi+1+\nu-\delta)^{(\nu-\delta-1)}}{(1-\eta \kappa(\xi, \delta)) \Gamma(\nu-\delta+1)}+T+2\right) \Delta y(0),
\end{aligned}
$$

we have

$$
\|y\|=\max \{y(T+2), \Delta y(0)\} \leq(T+2+m) \Delta y(0) .
$$

Therefore, $\alpha(y) \leq \psi(y)$ and $\|y\| \leq M \gamma(y)$ are satisfied, where $M=T+2+m$.

We now put growth condition on $f$ such that the boundary value problem (20)-(22) has at least three positive solutions belonging to the cone $P$. Then (1)-(2) has at least three positive solutions.

Theorem 11. Let $0<\beta, \delta \leq v<\varepsilon<1$, and suppose that (H1)-(H2) hold. In addition, assume that there exist positive numbers $k, a, b, d$ with $a<b \leq(h /(T+2)) d$ such that the following conditions are satisfied:

(H3) $f(t+v-1, u, v) \leq\left(1 / M_{1}\right) \varphi_{p}(d)$ for $(t, u, v) \in[0, T]_{\mathbb{N}_{0}} \times$ $[0,(T+2+m) d] \times[0,(T+2+m) d]$

(H4) $f(t+v-1, u, v)>\varphi_{p}\left((T+2) b / h M_{3}\right)$ for $(t, u, v) \epsilon$ $[h, T]_{\mathbb{N}_{h}} \times\left[\left((T+v-1)^{(v-1)} / \Gamma(v)\right) b,((T+2) / h) b\right] \times$ $[0,(T+2+m) d]$,

(H5) $f(t+v-1, u, v) \leq \min \{(p-1)(((\varepsilon-v) a \mid$ $\left.\left.\left(\sum_{i=0}^{T} q(i)\right)^{1 /(p-1)}+a /(N k)\right)^{p-2} a / N k, \varphi_{p}(a / N k)\right\}$ for $(t, u, v) \in[0, T]_{\mathbb{N}_{0}} \times[0, a] \times[0,(T+2+m) d]$,

(H6) $L=\left(\left(M_{2}+M_{3}\right) / N k\right)+\eta(\varepsilon-v) \sum_{s=0}^{\xi+1} \kappa(\xi-s-1, \delta)+$ $(\varepsilon-v)(T+2) \leq 1$.
Then FBVP (1)-(2) has at least three positive solutions $x_{1}, x_{2}$, and $x_{3}$ satisfying

$$
\begin{gathered}
x_{1}(t)=\Delta_{0}^{1-v} y_{1}(t), \\
x_{2}(t)=\Delta_{0}^{1-v} y_{2}(t), \\
x_{3}(t)=\Delta_{0}^{1-v} y_{3}(t), \\
\max _{t \in[0, T+1]_{N_{0}}}\left|\Delta y_{i}(t)\right| \leq d, \quad \text { for } i=1,2,3, \\
b<\min _{t \in[h, T+2]_{N_{h}}}\left|y_{1}(t)\right|, \\
\max _{t \in[0, T+2]_{N_{0}}}\left|y_{3}(t)\right|<a, \\
a<\max _{t \in[0, T+2]_{N_{0}}}\left|y_{2}(t)\right|, \\
\text { with } \min _{t \in[h, T+2]_{N_{h}}}\left|y_{2}(t)\right|<b .
\end{gathered}
$$

Proof. By the definition of operator $Q$ and its properties (i)(v), it suffices to show that the conditions of Lemma 4 hold with respect to $Q$.

Firstly, if $y \in \overline{P(\gamma, d)}$, we know $\gamma(y)=$ $\max _{t \in[0, T+1]_{N_{0}}}|\Delta y(t)| \leq d$. From (37), we have

$$
\max _{t \in[0, T+2]_{N_{0}}}|y(t)| \leq(T+2+m) d .
$$

Since $\kappa(t, \beta)$ is monotonic decreasing in variable $t$, then

$$
\begin{aligned}
0 & \leq{ }_{t+\nu-\beta} \Delta_{\nu-1}^{\beta} x(t) \\
& =\sum_{s=0}^{t} \kappa(t-s-2, \beta) \Delta y(s)+y(0) \kappa(t-1, \beta) \\
& =\sum_{s=1}^{t} \kappa(t-s-2, \beta) \Delta y(s)+y(1) \kappa(t-2, \beta) \\
& \quad-y(0)(\kappa(t-2, \beta)-\kappa(t-1, \beta)) \\
& \leq \sum_{s=2}^{t} \kappa(t-s-2, \beta) \Delta y(s)+y(2) \kappa(t-3, \beta) \\
& \quad-y(1)(\kappa(t-3, \beta)-\kappa(t-2, \beta)) \\
& \leq y(t+1) \leq(T+2+m) d,
\end{aligned}
$$




$$
\begin{aligned}
\Delta y & (T+1) \\
= & -\sum_{s=0}^{T} \kappa(T-s-1, \varepsilon) \Delta y(s) \\
& -\frac{\eta \kappa(T, \varepsilon)}{1-\eta \kappa(\xi, \delta)} \sum_{s=0}^{\xi+1} \kappa(\xi-s-1, \delta) \Delta y(s) \\
\leq & -\sum_{s=0}^{T} \kappa(T-s-1, \varepsilon) \\
& \left.-\frac{\eta \kappa(T, \varepsilon)}{1-\eta \kappa(\xi, \delta)} \sum_{s=0}^{\xi+1} \kappa(\xi-s-1, \delta)\right) d=\tau d .
\end{aligned}
$$

Thus, assumption (H3) and Lemma 10 imply that

$$
\begin{aligned}
& \gamma(Q y) \\
& =\max _{t \in[0, T+1]_{N_{0}}}|\Delta(Q y)(t)|=|\Delta(Q y)(0)| \\
& \quad=\varphi_{q}\left(\sum_{i=0}^{T} q(i) f\left(i+v-1, x(i+v-1),{ }_{i+\nu-\beta} \Delta_{\nu-1}^{\beta} x(i)\right)\right. \\
& \left.\quad+\varphi_{p}(\Delta y(T+1))\right) \\
& \quad=\varphi_{q}\left(\frac{1}{M_{1}} \varphi_{p}(d) \sum_{i=0}^{T} q(i)+\tau^{p-1} \varphi_{p}(d)\right) \\
& \left(\left(\frac{1}{M_{1}} \sum_{i=0}^{T} q(i)+\tau^{p-1}\right) \varphi_{p}(d)\right)=d .
\end{aligned}
$$

Hence, $Q: \overline{P(\gamma, d)} \rightarrow \overline{P(\gamma, d)}$.

To check condition $(S 1)$ of Lemma 4 , we choose $y_{0}(t)$ as follows for $t \in[0, T+2]_{\mathbb{N}_{0}}$ :

$y_{0}(t)$

$$
=\left\{\begin{array}{l}
-\frac{2 b}{(T+2)^{2}}(t-(T+2))^{2}+\frac{T+2}{h} b, \quad t \text { is even; } \\
-\frac{2 b}{(T+3)^{2}}(t-(T+2))^{2}+\frac{T+2}{h} b, \quad t \text { is odd. }
\end{array}\right.
$$

It is easy to verify that $y_{0} \in P(\gamma, \theta, \alpha, b,((T+2) / h) b, d)$ and $\alpha\left(y_{0}\right)=\min _{t \in[h, T+2]_{\mathbb{N}_{h}}}\left|y_{0}(t)\right|=y_{0}(h)>b$. So $\{y \in$ $P(\gamma, \theta, \alpha, b,((T+2) / h) b, d) \mid \alpha(y)>b\} \neq \emptyset$.

Therefore, if $y \in P(\gamma, \theta, \alpha, b,((T+2) / h) b, d)$, then $b \leq$ $y(t) \leq((T+2) / h) b,|\Delta y(t)| \leq d$ for $t \in[h, T+2]_{\mathbb{N}_{h}}$.
By (27), we have $(T+v-1)^{(v-1)} b / \Gamma(\nu) \leq x(t+v-1) \leq$ $((T+2) / h) b$. According to assumption $(H 4)$ and Lemma 10, we get

$$
\begin{aligned}
& \alpha(Q y) \\
& =\min _{t \in[h, T+2]_{\mathbb{N}_{h}}}|(Q y)(t)| \geq \frac{h}{T+2} \max _{t \in[0, T+1]_{\mathbb{N}_{0}}}|(Q y)(t)| \\
& =\frac{h}{T+2}\left(\frac{\eta}{1-\eta \kappa(\xi, \delta)} \sum_{s=0}^{\xi+1} \kappa(\xi-s-1, \delta) \varphi_{q}\right. \\
& {\left[\sum_{j=s}^{T} q(j) f(j+v-1\right.} \\
& \left.x(j+v-1),{ }_{j+\nu-\beta} \Delta_{\nu-1}^{\beta} x(j)\right) \\
& \left.+\varphi_{p}(\Delta y(T+1))\right] \\
& +\sum_{i=0}^{T+1}\left[\varphi _ { q } \left(\sum_{j=i}^{T} q(j) f(j+v-1, x(j+v-1),\right.\right. \\
& \left.{ }_{j+\nu-\beta} \Delta_{\nu-1}^{\beta} x(j)\right) \\
& \left.\left.\left.+\varphi_{p}(\Delta y(T+1))\right)\right]\right) \\
& \geq \frac{h}{T+2} \sum_{i=0}^{T} \varphi_{q}\left[\sum_{j=i}^{T} q(j)\right. \\
& f(j+v-1, x(j+v-1), \\
& \left.\left.{ }_{j+\nu-\beta} \Delta_{\nu-1}^{\beta} x(j)\right)\right] \\
& \geq \frac{h}{T+2} \sum_{i=0}^{T} \varphi_{q}\left[\sum_{j=i}^{T} q(j) \varphi_{p}\left(\frac{(T+2) b}{h M_{3}}\right)\right]=b \text {. }
\end{aligned}
$$

This shows that condition (S1) of Lemma 4 is satisfied. Secondly, from (35), we have

$$
\alpha(Q y) \geq \frac{h}{T+2} \theta(Q y)>b
$$

for all $y \in P(\gamma, \alpha, b, d)$ with $\theta(Q y)>((T+2) / h) b$. Thus, condition (S2) of Lemma 4 is satisfied.

We finally exhibit that (S3) of Lemma 4 is also satisfied. Clearly, as $\psi(0)=0<a$, we have $0 \notin R(\gamma, \psi, a, d)$. Suppose 
that $y \in R(\gamma, \psi, a, d)$ with $\psi(y)=a$. Since $\kappa(t, \varepsilon)$ is monotonic increasing in variable $t$, we have

$$
\begin{aligned}
\Delta y(T+1) & \\
= & -\sum_{s=0}^{T} \kappa(T-s-1, \varepsilon) \Delta y(s)-y(0) \kappa(T, \varepsilon) \\
= & -\sum_{s=1}^{T} \kappa(T-s-1, \varepsilon) \Delta y(s)-y(1) \kappa(T-1, \varepsilon) \\
& +(\kappa(T-1, \varepsilon)-\kappa(T, \varepsilon)) y(0) \\
\leq & -\sum_{s=1}^{T} \kappa(T-s-1, \varepsilon) \Delta y(s)-y(1) \kappa(T-1, \varepsilon) \\
\leq & -\sum_{s=2}^{T} \kappa(T-s-1, \varepsilon) \Delta y(s)-y(2) \kappa(T-2, \varepsilon) \\
\leq & \ldots \\
\leq & -\kappa(-1, \varepsilon) y(T+1) \leq(\varepsilon-\nu) a .
\end{aligned}
$$

Next, we consider two cases.

Case i. Consider $p \geq 2$. By (H5) and the inequality $x^{p-1}+$ $y^{p-1} \leq(x+y)^{p-1}$ for $x, y \in \mathbb{R}^{+}$, we can obtain that

$$
\begin{aligned}
\psi(Q y) & \max _{t \in[0, T+2]_{N_{0}}}|(Q y)(t)| \\
\leq & \frac{\eta}{1-\eta \kappa(\xi, \delta)} \sum_{s=0}^{\xi+1} \kappa(\xi-s-1, \delta) \varphi_{q} \\
& \cdot\left(\sum_{j=s}^{T} q(j) f\left(j+\nu-1, x(j+\nu-1),{ }_{j+\nu-\beta} \Delta_{\nu-1}^{\beta} x(j)\right)\right. \\
& \left.+\varphi_{p}((\varepsilon-\nu) a)\right) \\
& +\sum_{i=0}^{T+1} \varphi_{q}\left(\sum_{j=i}^{T} q(j) f(j+\nu-1, x(j+\nu-1),\right. \\
\leq & \frac{\eta}{1-\eta \kappa(\xi, \delta)} \sum_{s=0}^{\xi+1} \kappa(\xi-s-1, \delta) \varphi_{q} \\
& \cdot\left(\sum_{j=s}^{T} q(j) \varphi_{p}\left(\frac{a}{N k}\right)+\varphi_{p}((\varepsilon-\nu) a)\right) \\
+\varphi_{p}((\varepsilon-\nu) a) & \left.\Delta_{\nu-1}^{\beta} x(j)\right)
\end{aligned}
$$

Case ii. Consider $1<p<2$. By (H5) (H6) and the inequality $(x+y)^{p-1}-y^{p-1} \geq(p-1)(x+y)^{p-2} x$, for $x, y \in \mathbb{R}^{+}$, we can have

$$
\begin{aligned}
& \psi(Q y) \\
& =\max _{t \in[0, T+2]_{N_{0}}}|(Q y)(t)| \\
& \leq \frac{\eta}{1-\eta \kappa(\xi, \delta)} \sum_{s=0}^{\xi+1} \kappa(\xi-s-1, \delta) \\
& \cdot\left(\sum_{j=s}^{T} q(j)\right)^{1 /(p-1)} \varphi_{q}(f(j+\nu-1, x(j+\nu-1), \\
& \left.+\sum_{i=0}^{T+1}\left(\sum_{j=i}^{T} q(j)\right)^{\beta} x(j)\right) \\
& \left.+\varphi_{p}((\varepsilon-\nu) a) \frac{1}{\sum_{j=s}^{T} q(j)}\right) \\
& \varphi_{q}(f(j+\nu-1, x(j+\nu-1), \\
& \left.j+\nu-\beta \Delta_{\nu-1}^{\beta} x(j)\right)
\end{aligned}
$$




$$
\begin{aligned}
& \left.+\varphi_{p}((\varepsilon-v) a) \frac{1}{\sum_{j=i}^{T} q(j)}\right) \\
& \leq \frac{a \eta}{1-\eta \kappa(\xi, \delta)} \sum_{s=0}^{\xi+1} \kappa(\xi-s-1, \delta)\left(\sum_{j=s}^{T} q(j)\right)^{1 /(p-1)} \\
& \cdot \varphi_{q}\left((p-1)\left(\frac{(\varepsilon-v)}{\left(\sum_{j=s}^{T} q(j)\right)^{1 /(p-1)}}+\frac{1}{N k}\right)^{p-2}\right. \\
& \left.\cdot \frac{1}{N k}+\varphi_{p}\left(\frac{(\varepsilon-\nu)}{\left(\sum_{j=s}^{T} q(j)\right)^{1 /(p-1)}}\right)\right) \\
& +a \sum_{i=0}^{T+1}\left(\sum_{j=i}^{T} q(j)\right)^{1 /(p-1)} \\
& \cdot \varphi_{q}\left((p-1)\left(\frac{(\varepsilon-\nu)}{\left(\sum_{j=i}^{T} q(j)\right)^{1 /(p-1)}}+\frac{1}{N k}\right)^{p-2}\right. \\
& \left.\cdot \frac{1}{N k}+\varphi_{p}\left(\frac{(\varepsilon-v)}{\left(\sum_{j=i}^{T} q(j)\right)^{1 /(p-1)}}\right)\right) \\
& \leq \frac{a \eta}{1-\eta \kappa(\xi, \delta)} \sum_{s=0}^{\xi+1} \kappa(\xi-s-1, \delta) \\
& \cdot\left(\sum_{j=s}^{T} q(j)\right)^{1 /(p-1)}\left(\frac{(\varepsilon-v)}{\left(\sum_{j=s}^{T} q(j)\right)^{1 /(p-1)}}+\frac{1}{N k}\right) \\
& +a \sum_{i=0}^{T+1}\left(\sum_{j=i}^{T} q(j)\right)^{1 /(p-1)}\left(\frac{(\varepsilon-\nu)}{\left(\sum_{j=i}^{T} q(j)\right)^{1 /(p-1)}}+\frac{1}{N k}\right) \\
& \leq \frac{a \eta}{(1-\eta \kappa(\xi, \delta)) N k} \sum_{s=0}^{\xi+1} \kappa(\xi-s-1, \delta)\left(\sum_{j=s}^{T} q(j)\right)^{1 /(p-1)} \\
& +\frac{a \eta(\varepsilon-\nu)}{1-\eta \kappa(\xi, \delta)} \sum_{s=0}^{\xi+1} \kappa(\xi-s-1, \delta)+(\varepsilon-\nu)(T+2) a \\
& +\frac{a}{N k} \sum_{i=0}^{T+1}\left(\sum_{j=i}^{T} q(j)\right)^{1 /(p-1)} \\
& \leq \frac{a}{N k} M_{2}+a \eta(\varepsilon-\nu) \sum_{s=0}^{\xi+1} \kappa(\xi-s-1, \delta) \\
& +(\varepsilon-v)(T+2) a+\frac{a}{N k} M_{3} \\
& =L a \leq a \text {. }
\end{aligned}
$$

So, condition (S3) of Lemma 4 is satisfied. Therefore, Lemma 4 implies that the FBVP (20)-(22) has at least three positive solutions $y_{1}, y_{2}$, and $y_{3}$ satisfying (38); that is, the FBVP (1)-(2) has at least three positive solutions $x_{1}, x_{2}$, and $x_{3}$ satisfying (38). The proof is complete.

Theorem 12. Let $\delta \leq v=\varepsilon$ and $(H 1)-(H 4)$ hold. In addition, assume that the following condition is satisfied:

(H7) $f \leq \varphi_{p}\left(a /\left(M_{2}+M_{3}\right)\right)$.

Then the FBVP (1)-(2) has at least three positive solutions $x_{1}, x_{2}$, and $x_{3}$ satisfying (38).

Proof. If $\varepsilon=v,(21)$ is equivalent to $\Delta y(T+1)=0$ by (14). Similar to discussion in Theorem 11, we know that (24) holds. For $t \in[0, T+2]_{\mathbb{N}_{0}}$, define an operator $Q: P \rightarrow B$ by

$$
\begin{aligned}
& \text { Qy }(t) \\
& =\frac{\eta}{1-\eta \kappa(\xi, \delta)} \sum_{s=0}^{\xi+1} \kappa(\xi-s-1, \delta) \varphi_{q} \\
& \cdot\left(\sum_{j=s}^{T} q(j)\right. \\
& \left.\cdot f\left(j+v-1, x(j+v-1),{ }_{j+\nu-\beta} \Delta_{\nu-1}^{\beta} x(j)\right)\right) \\
& +\sum_{i=0}^{t-1}\left(\varphi _ { q } \left(\sum_{j=i}^{T} q(j)\right.\right. \\
& \left.\left.\cdot f\left(j+v-1, x(j+v-1),{ }_{j+\nu-\beta} \Delta_{\nu-1}^{\beta} x(j)\right)\right)\right) .
\end{aligned}
$$

The rest of the proof is similar to Theorem 11, so it is omitted. The proof is complete.

Example 13. Consider the boundary value problem:

$$
\begin{gathered}
\Delta\left(\varphi_{p}\left(\Delta_{-0.05}^{0.95} x(t)\right)\right) \\
+f\left(t+v-1, x(t+v-1),{ }_{t+0.05} \Delta_{-0.05}^{0.9} x(t)\right) \\
=0, \quad t \in[0,10]_{\mathbb{N}_{0}}, \\
{\left[\Delta_{-0.05}^{0.96} x(t)\right]_{t=10.99}=0,} \\
x(-0.05)=\frac{1}{2}\left[\Delta_{-0.05}^{0.94} x(t)\right]_{t=2.01} .
\end{gathered}
$$

Compared with (1), we have $q(t)=1, p=3, q=3 / 2, \xi=1$, $T=10, \nu=0.95, \beta=0.9, \varepsilon=0.96, \delta=0.94, \eta=1 / 2, h=6$, and

$$
\begin{aligned}
& f(t+v-1, u, v) \\
& =\left\{\begin{array}{cl}
|\sin (t+v-1)|+49+\frac{v}{1500}, & 0 \leq u \leq 500 ; \\
|\sin (t+v-1)|+49 & \\
\quad+\frac{1}{11.5}(u-500)^{2}+\frac{v}{1500}, & 500 \leq u \leq 1000 \\
|\sin (t+v-1)|+49 & \\
+\frac{1}{11.5}(500)^{2}+\frac{v}{1500}, & u \geq 1000 .
\end{array}\right.
\end{aligned}
$$

Then, FBVP (49) has at least three positive solutions. 
Proof. Choose $a=500, b=1800, d=5000, p=3, q=3 / 2$, and $k=2$.

By computation, we know $\tau \approx 0.0021, M_{1} \approx 11, M_{2} \approx$ $1.56, M_{3}=\sum_{j=0}^{10}(11-j)^{1 / 2} \approx 25.78, N=M_{3}$, and $m \approx 0.52$.

It is easy to see that $0<a<b<2 d$. And $f$ satisfies that

$$
\begin{aligned}
& f(t+v-1, u, v) \\
& \quad \leq 50+\frac{250000}{11.5}+\frac{62590.21}{1500} \approx 21830.21 \\
& \quad<2270663.03 \approx \frac{1}{M_{1}} \varphi_{p}(d),
\end{aligned}
$$

for $(t, u, v) \in[0,10]_{\mathbb{N}_{0}} \times[0,62590.21] \times[0,62590.21]$;

$$
\begin{aligned}
& f(t+v-1, u, v) \\
& \geq 49+\frac{500^{2}}{11.5} \approx 21788>19488 \\
& \approx\left(\frac{3600}{25.78}\right)^{2}=\varphi_{p}\left(\frac{(T+2) b}{h M_{3}}\right),
\end{aligned}
$$

for $(t, u, v) \in[6,10]_{\mathbb{N}_{0}} \times[1800 \Gamma(10+v) / \Gamma(11) \Gamma(\nu), 3600] \times$ $[0,62590.21]$;

$$
\begin{aligned}
f(t+v-1, u, v) & <50+\frac{62590.21}{1500} \approx 91.73 \\
& \leq\left(\frac{500}{2 \times 25.78}\right)^{2}<94.04 \approx \varphi_{p}\left(\frac{a}{2 N}\right),
\end{aligned}
$$

for $(t, u, v) \in[0,10]_{\mathbb{N}_{0}} \times[0,500] \times[0,65000]$. Consider

$$
\begin{aligned}
L & =\frac{1.56+25.78}{2 \times 25.78}+\frac{1}{2} \times \frac{2.01 \times 1.01 \times 0.01}{2}+12 \times 0.01 \\
& \approx 0.65<1 .
\end{aligned}
$$

Thus, the conditions of Theorem 11 are satisfied. Therefore, the FBVP (49) has at least three positive solutions satisfying

$$
\begin{aligned}
& x_{1}(t)=\Delta_{0}^{0.05} y_{1}(t), \\
& x_{2}(t)=\Delta_{0}^{0.05} y_{2}(t), \\
& x_{3}(t)=\Delta_{0}^{0.05} y_{3}(t) \text {, } \\
& \max _{t \in[0,11]_{\mathbb{N}_{0}}}\left|\Delta y_{i}(t)\right| \leq 5000, \quad \text { for } i=1,2,3, \\
& 1800<\min _{t \in[6,12]_{\mathbb{N}_{6}}}\left|y_{1}(t)\right|, \\
& \max _{t \in[0,12]_{N_{0}}}\left|y_{3}(t)\right|<500, \\
& 500<\max _{t \in[0,12]_{\mathbb{N}_{0}}}\left|y_{2}(t)\right| \\
& \text { with } \min _{t \in[6,12]_{\mathbb{N}_{6}}}\left|y_{2}(t)\right|<1800 \text {. }
\end{aligned}
$$

\section{Conflict of Interests}

The authors declare that there is no conflict of interests regarding the publication of this paper.

\section{Acknowledgment}

The Project was supported by the National Natural Science Foundation of China (11161049).

\section{References}

[1] E. Zeidler, Nonlinear Functional Analysis and Its Applications I: Fixed-Point Theorems, Springer, New York, NY, USA, 1993.

[2] R. P. Agarwal, D. O'Regan, and P. J. Y. Vong, Positive Solutions of Differential, Difference and Integral Equations, Kluwer Academic, Dordrecht, The Netherlands, 1999.

[3] R. I. Avery and J. Henderson, "Three symmetric positive solutions for a second-order boundary value problem," Applied Mathematics Letters, vol. 13, no. 3, pp. 1-7, 2000.

[4] R. I. Avery and A. C. Peterson, "Three positive fixed points of nonlinear operators on ordered Banach spaces," Computers \& Mathematics with Applications, vol. 42, no. 3-5, pp. 313-322, 2001.

[5] J. Henderson and H. B. Thompson, "Multiple symmetric positive solutions for a second order boundary value problem," Proceedings of the American Mathematical Society, vol. 128, no. 8, pp. 2373-2379, 2000

[6] E. R. Kaufmann, "Multiple positive solutions for higher order boundary value problems," The Rocky Mountain Journal of Mathematics, vol. 28, no. 3, pp. 1017-1028, 1998.

[7] R. W. Leggett and L. R. Williams, "Multiple positive fixed points of nonlinear operators on ordered Banach spaces," Indiana University Mathematics Journal, vol. 28, no. 4, pp. 673-688, 1979.

[8] R. I. Avery, "A generalization of the Leggett-Williams fixed point theorem," Mathematical Sciences Research Hot-Line, vol. 3, no. 7, pp. 9-14, 1999.

[9] F. M. Atici and P. W. Eloe, "A transform method in discrete fractional calculus," International Journal of Difference Equations, vol. 2, no. 2, pp. 165-176, 2007.

[10] C. S. Goodrich, "Solutions to a discrete right-focal fractional boundary value problem," International Journal of Difference Equations, vol. 5, no. 2, pp. 195-216, 2010.

[11] C. S. Goodrich, "Existence of a positive solution to a first-order p-Laplacian BVP on a time scale," Nonlinear Analysis: Theory, Methods \& Applications, vol. 74, no. 5, pp. 1926-1936, 2011.

[12] F. M. Atıcı and P. W. Eloe, “Two-point boundary value problems for finite fractional difference equations," Journal of Difference Equations and Applications, vol. 17, no. 4, pp. 445-456, 2011.

[13] F. M. Atici and P. W. Eloe, "Discrete fractional calculus with the nabla operator," Electronic Journal of Qualitative Differential Equations I, no. 3, pp. 1-12, 2009.

[14] F. M. Atici and S. Şengül, "Modeling with fractional difference equations," Journal of Mathematical Analysis and Applications, vol. 369, no. 1, pp. 1-9, 2010.

[15] C. S. Goodrich, "Existence and uniqueness of solutions to a fractional difference equation with nonlocal conditions," Computers \& Mathematics with Applications, vol. 61, no. 2, pp. 191-202, 2011. 
[16] M. T. Holm, The Theory of Discrete Fractional Calculus: Development and Application, DigitalCommons@University of Nebraska-Lincoln, 2011.

[17] F. M. Atici and P. W. Eloe, "Linear systems of fractional nabla difference equations," The Rocky Mountain Journal of Mathematics, vol. 41, no. 2, pp. 353-370, 2011.

[18] R. A. Ferreira, "A discrete fractional Gronwall inequality," Proceedings of the American Mathematical Society, vol. 140, no. 5, pp. 1605-1612, 2012.

[19] R. A. C. Ferreira, "Existence and uniqueness of solution to some discrete fractional boundary value problems of order less than one," Journal of Difference Equations and Applications, vol. 19, no. 5, pp. 712-718, 2013.

[20] R. A. Ferreira and C. S. Goodrich, "Positive solution for a discrete fractional periodic boundary value problem," Dynamics of Continuous, Discrete \& Impulsive Systems. Series A. Mathematical Analysis, vol. 19, no. 5, pp. 545-557, 2012.

[21] C. S. Goodrich, "Continuity of solutions to discrete fractional initial value problems," Computers \& Mathematics with Applications, vol. 59, no. 11, pp. 3489-3499, 2010.

[22] C. S. Goodrich, "Existence of a positive solution to a system of discrete fractional boundary value problems," Applied Mathematics and Computation, vol. 217, no. 9, pp. 4740-4753, 2011.

[23] C. S. Goodrich, "On a discrete fractional three-point boundary value problem," Journal of Difference Equations and Applications, vol. 18, no. 3, pp. 397-415, 2012.

[24] C. S. Goodrich, "On a fractional boundary value problem with fractional boundary conditions," Applied Mathematics Letters, vol. 25, no. 8, pp. 1101-1105, 2012.

[25] C. S. Goodrich, "On a first-order semipositone discrete fractional boundary value problem," Archiv der Mathematik, vol. 99, no. 6, pp. 509-518, 2012.

[26] C. S. Goodrich, "On semipositone discrete fractional boundary value problems with non-local boundary conditions," Journal of Difference Equations and Applications, vol. 19, no. 11, pp. 17581780, 2013.

[27] C. S. Goodrich, "On discrete sequential fractional boundary value problems," Journal of Mathematical Analysis and Applications, vol. 385, no. 1, pp. 111-124, 2012.

[28] Z. Huang and C. Hou, "Solvability of nonlocal fractional boundary value problems," Discrete Dynamics in Nature and Society, vol. 2013, Article ID 943961, 9 pages, 2013.

[29] Z. Xie, Y. Jin, and C. Hou, "Multiple solutions for a fractional difference boundary value problem via variational approach," Abstract and Applied Analysis, vol. 2012, Article ID 143914, 16 pages, 2012. 


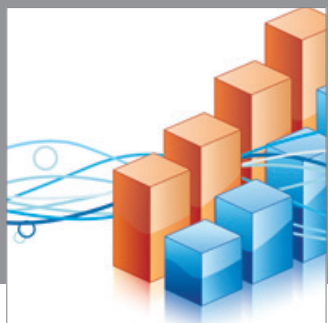

Advances in

Operations Research

mansans

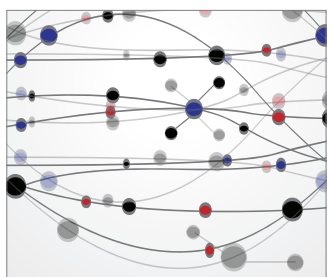

The Scientific World Journal
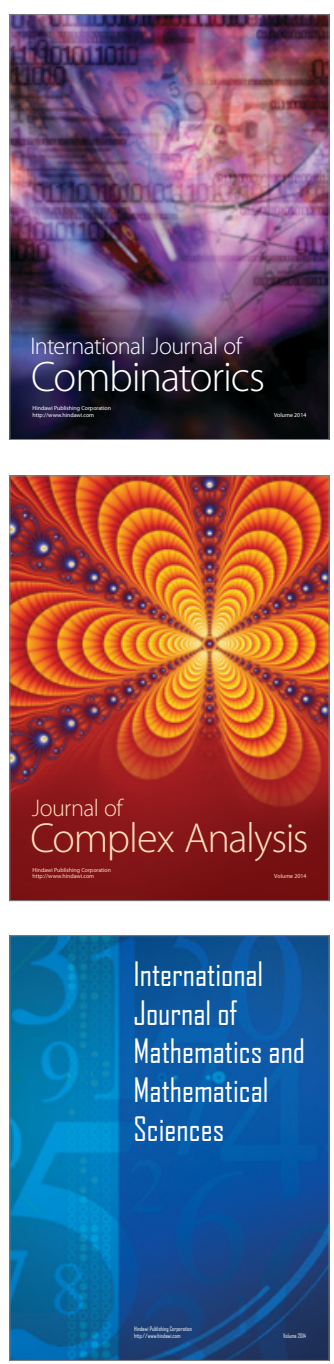
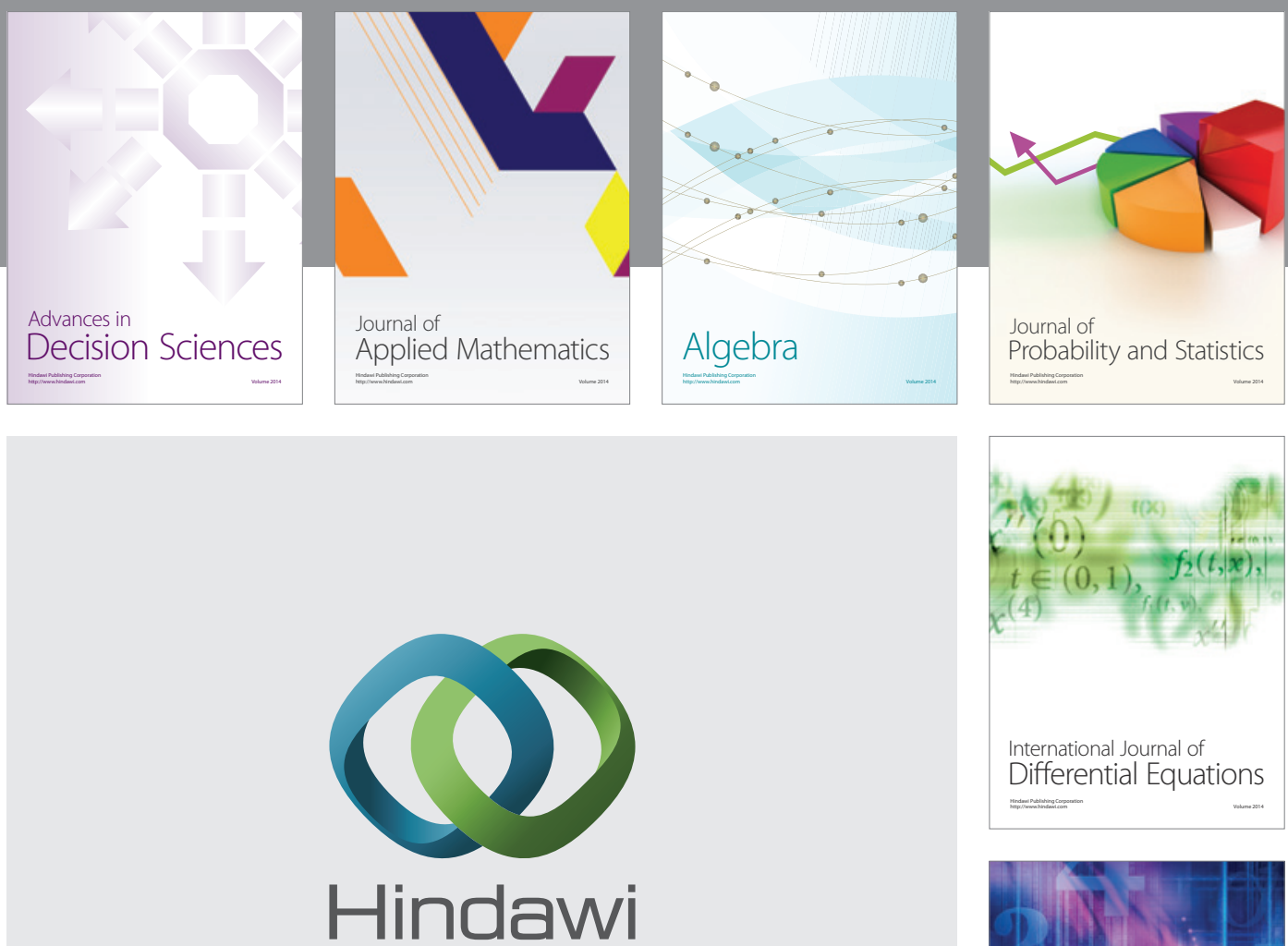

Submit your manuscripts at http://www.hindawi.com
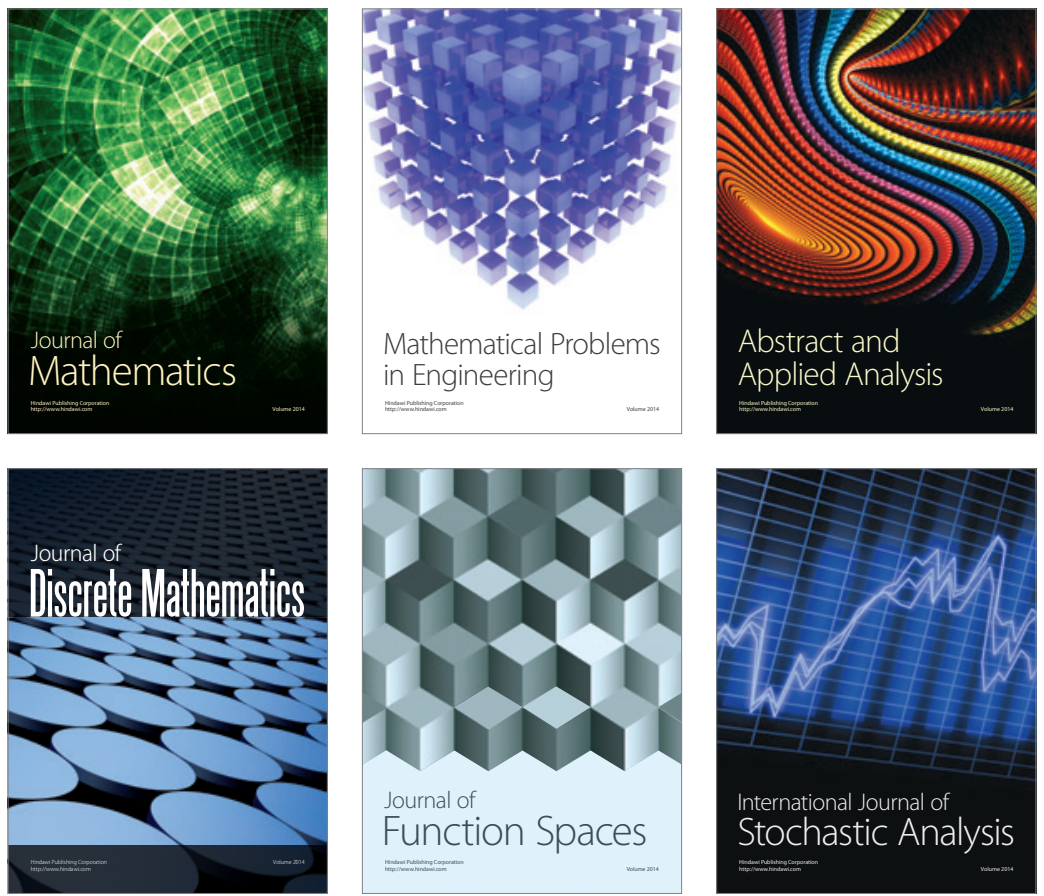

Journal of

Function Spaces

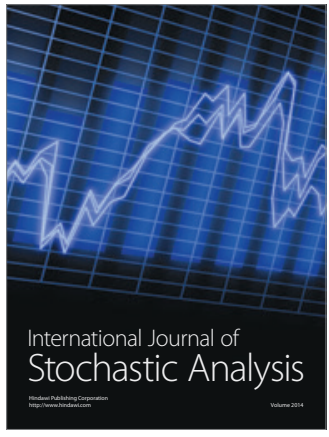

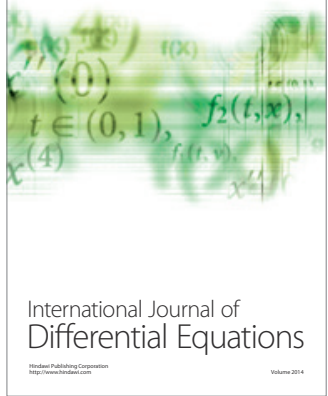
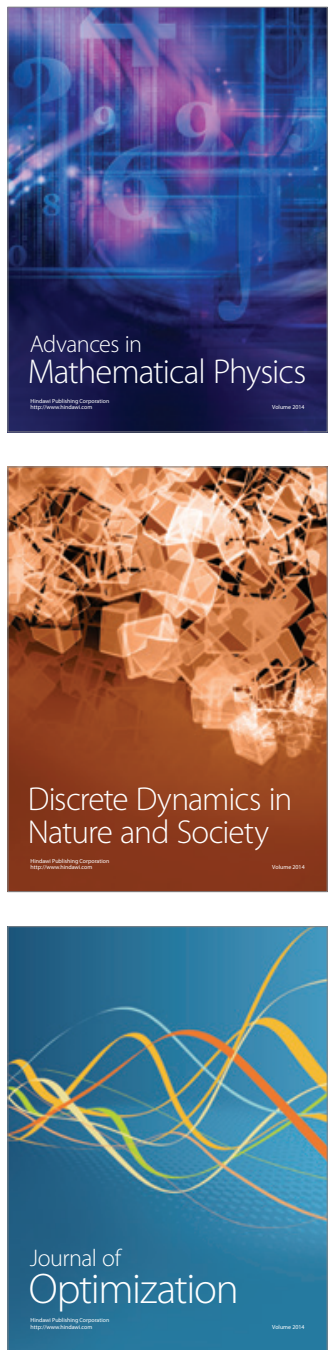